\title{
A physical basis for the precise location of the division site of rod-shaped bacteria: the Central Stress Model
}

\author{
Arthur L. Koch† and Joachim-Volker Höltje \\ Author for correspondence: Arthur L. Koch. Tel: +1 812855 5036. Fax: +1 8128556705. \\ e-mail: Koch@Indiana.edu
}

Max-Planck-Institut für Entwicklungsbiologie, Abteilung Biochemie, Spemannstrasse 35, D-72076, Tübingen, Germany
There are difficulties with all published models that attempt to explain how rod-shaped bacteria locate their midpoint in preparation for the next cell division. Many bacteria find their middle quite accurately. This evenness of partition has been measured cytologically and is implied by the persistence of synchrony under certain circumstances. Previously, a number of models for the control of cell division have been proposed based on aspects of molecular genetics, ultrastructure measurements, or biochemical kinetics. This paper points out that none of the models in spite of their quite different natures can explain the precision of the location of the division site. Here, the 'Central Stress Model' is proposed which depends on the partition of wall tension between the cytoplasmic membrane (CM) and the murein layer in such a way that the $\mathrm{CM}$ at the centre of the rod experiences a higher stress than near the poles and that this peak stress increases through the cell cycle. The model assumes that: (i) murein is not incorporated at an established pole but is incorporated diffusely over the sidewall and intensely at sites of cell constriction; (ii) CM is synthesized over the entire cell wall; (iii) the murein and CM layers are attached non-covalently to each other, and interact physically with each other; (iv) this differential location of synthesis leads to a 'tug-ofwar' that creates differential stresses that peak at the cell centre. Because of the fluid nature of the phospholipid bilayer there is a flux of lipid from the established poles towards the cell centre as the murein sidewall elongates. The flux from the pole lowers the tension in the $\mathrm{CM}$ at the ends of the sidewalls and creates a peak tension in the centre. Cells also have a discontinuity in the stresses in the murein at the junction of the curved pole with the cylindrical region of the cell wall (a doubling of the hoop stress above the axial stress). Thus in addition to the midpoint of the cell, these junctions between the polar caps and the cylindrical part of the cell wall are characterized by an abrupt change in the surface stress and we suggest that this can trigger cell division at these junctions to form a chromosome-less minicell. Two other assumptions of the model are that the cell has a membrane-associated system to sense the stress, and to trigger cell division locally when a threshold has been reached. It is suggested that there is a special two-component sensory system responding to tension in the CM. As the cell cycle progresses, and the stress in the centre of the cell exceeds some threshold, a system molecule triggers a cell division event at that site. Like other two-component systems, the sensory component of this two-component system is assumed to be distributed all over the CM. It is also assumed that when the sensory component is triggered it also causes local changes that ensure that division occurs at that site. Consequently, this model can explain why sister cells have very nearly the same size (length, 


\section{volume, or biomass) and why genes that control a mechanism that senses cell size and initiates cell division have never been identified because they may not exist.}

Keywords: cell division mechanisms, differential stresses, coupling of wall layers, Central Stress Model, division site location

\section{INTRODUCTION}

The rod-shaped cells of the bacteria Escherichia coli (Trueba, 1981) and Bacillus subtilis (Burdett \& Higgins, 1978) divide very precisely at their midpoints to produce daughters of nearly equal size. The coefficient of variation (CV) of sister lengths has been observed to be as low as $4 \%$. Since random variations for different steps combine with each other to give a larger total variation, the inherent precision of the initiating event, if measurement errors could be eliminated, might have a $\mathrm{CV}$ considerably less than $4 \%$. Thus, a significant and well-documented fact in bacterial physiology is that cell division can be highly precise. Koch et al. (1981) elaborated on the importance of the unexpected accuracy of cell division and reviewed the literature. At present, how the cells can achieve this precision or how they even approximately fix the place where the next division is to take place are only matters for speculation among microbial physiologists because there are no direct experiments. Other prokaryotes may be quite imprecise in partitioning at cell division. Additionally, certain mutants exist in which division occasionally takes place at one of the poles instead of the middle and pinches off DNA-less spherical minicells (Adler et al., 1967). Thus, supplementary to the midpoint of the cell, the poles/sidewall junction of the pole with the cylindrical part is a potential site for cell division to take place. The genetic evidence is that normally the polar sites are prevented from being used by the minCD cell division control system (de Boer et al., 1989). The question of where the next cell division site will be located and when cell division will be initiated are important aspects of prokaryote physiology. At present there is little evidence from genetics and the major concept is the old observation (reviewed below) that the Gram-negative cell seems to divide when it has a critical mass and not a critical age.

It was this unsatisfactory state of knowledge, particularly that the cell division could, but need not, bisect the cell precisely that led to the Central Stress Model proposed here. It assumes that: (i) murein (peptidoglycan) is not synthesized at an established pole; (ii) that cytoplasmic membrane $(\mathrm{CM})$ is synthesized over the entire cell wall; (iii) the murein and CM layers are attached non-covalently to each other; (iv) this differential location of synthesis leads to a 'tug-of-war' that creates differential stresses that peak at the cell centre; (v) the cell has a system to sense the stress; (vi) cell division is triggered when a threshold has been reached.

A number of models have been put forward to explain the positioning of the site of division. All of these models are incomplete, however, since most fail to explain how cell division occurs exactly at the centre of the mother cell and one model assumes a cellular mechanism for which there is no precedence. Therefore for this key process in prokaryote biology we propose another basis; i.e. during growth the mechanical stress in the wall created by the interaction of the CM with the murein layer under special conditions leads to a sufficiently high value of tension in the CM in the centre of the cell to locally trigger division. If this proposal is correct, then one implication is that one would not be able to find a gene that locates the septal site. No such gene has been found, and we suggest that no such gene exists.

\section{Current models}

We shall review four models for the control of cell division and point out why they are inadequate.

(i). The periseptal annuli (PSA) model of MacAlister $e t$ al. (1983) postulates that a pair of periseptal annuli are created on either side of an incipient division site. The new annuli then move during further growth of the cell to sites that are one-quarter and three-quarters of the cell's length. After this repositioning, they remain at the same relative positions as the cell continues to elongate. After the next cell division, each automatically becomes the midpoint position of the daughter cell and thereby establishes the site of the next cell division event (Cook et al., 1986, 1987, 1989; Rothfield et al., 1991). The fundamental criticism of the PSA model as a mechanism for division site selection is that it is not a full model because it does not attempt to consider how these annuli, or the plasmolysis 'bays' that are presumed to mark them, can 'know' when to stop moving. Only with the addition of other logical elements (perhaps derived from the model presented here) to spatially define the sites could the PSA model be a part of an adequate model for cell division. It must be mentioned that there currently is a controversy about this model, with Cook \& Rothfield (1994a, b) supporting it and Van Helvoort et al. (1994) and Woldringh (1994) against the hypothesis that the location where plasmolysis takes place moves from near a previous site to where the next division is to take place.

(ii). The nucleoid occlusion model (Mulder \& Woldringh, 1989, 1993; Woldringh et al., 1991) proposes that the nucleoid produces a signal that inhibits cell division by blocking murein synthesis. This model is a complex one with many additional elements, but it is an incomplete model because it does not specify how the nucleoids 'know' when and how far to move from each other after the sister nuclei have become separated. The length of the observable nucleoid does not appear to be very constant. 
Additionally, because the model has been formulated to explain the localization of cell division between the nucleoids, it does not explain the experimental finding that the cell divides very near the middle of its length from pole to pole. Other objections to this model are raised by Cook \& Rothfield (1994a, b).

(iii). The renovated replicon model (Koch et al., 1981; Koch, 1988b, 1990) is in principle capable of the observed precision. The model is based on the fact that the replicating bacterial chromosome develops into a symmetrical 'theta' structure during DNA replication. If the origin regions attached to the opposite ends of the cell and terminus region became attached to the replisome apparatus that replicates the chromosome, the growing DNA structure could develop forces that would centre the replisome, which is presumed to be loosely attached to the cell envelope, by sliding this structure upon the CM to equalize the stresses to reach the exact midpoint between the two poles. For the model to function, the two origins of the replicating chromosomes need to be physically attached to the opposite pole-tips for at least part of the cell cycle. However, there is evidence that the origin region of the chromosome only binds with high affinity to the wall for a short time after synthesis (Ogden et al., 1988). Although several adjustments of the model could be made, it is also incomplete because it does not explain the precise re-binding to the polar regions so that these loci can serve to centre the replisome with the tension developed in the DNA theta structure.

(iv). The activator-depletion substrate model (Meinhardt, 1982) is a special variant of a more general biochemical theory that describes pattern formation by an autocatalytic system that produces a rapidly diffusing inhibitor. Accordingly, particular classes of metabolic processes functioning within a confined space, i.e. a container, such as the cell itself, could generate spatial maxima and minima of concentrations along its length through enzymic action. These processes have the property of creating spatial gradients where there were none before and in principle could define the midpoint of a cell given a number of additional special circumstances such as appropriate and very different diffusion constants for the substrate and activator. This is important because the model was generated to cover a larger scale than the 1-3 $\mu \mathrm{m}$ length of bacterial cells. This model would be attractive if the hypothesis were enlarged to explain how the system regenerates itself at the end of each cell cycle. For the mechanism to work generation after generation, it is necessary at each new generation to recreate a uniform substrate concentration throughout the cell and destroy all of the activator from the previous cycle. Additionally, because the kinetic system produces a broad optimum, the model as it now stands may not account for the observed exactness of cell division.

\section{Role of mechanical forces in locating the geometric centre of a system}

Since cell division is an essential feature of even the leastdeveloped living systems, one may argue that its basis is likely to be a quite simple principle. A simple imprecise mechanism, no doubt, was all that was available early in the history of life (Koch, 1985, 1995). Subsequently, living forms acquired additional control features to make cell division more precise and better correlated with pertinent cellular events. For E. coli and B. subtilis, the control seems to be much more accurate than it need be, so we therefore asked whether the basis of location in rodshaped bacteria could be the simple physics of mechanical forces. Such forces should be inherently very precise and could function in dividing the cell at its geometric middle. We believe that the multilayered wall structure of bacteria with their external sacculus provides a basis for the action of physical forces.

Distribution of stresses. Consider a planar structure being subject to tension generated from two points at its ends, indicated by the two large arrowheads in Fig. 1(a). The stresses resulting from these forces pulling against each other generate tension between them and will be of uniform intensity and have uniform physical properties throughout the intervening region as shown by the shorter double-headed arrows. Evidently, the higher the force the higher will be the tension. With sufficiently high tension, the structure may fail and tear in two. This will happen at the point where there is an imperfection or defect in the structure (Fig. 1a, b) according to the dictum 'a chain is as strong as its weakest link'. With opposing forces applied only at two ends and no defects, failure would occur anywhere along the length of the structure and be dependent on structural abnormalities (or for a bacterium, bombardment by Brownian motion, etc.). This 'weakest link' idea is actually the key principle of

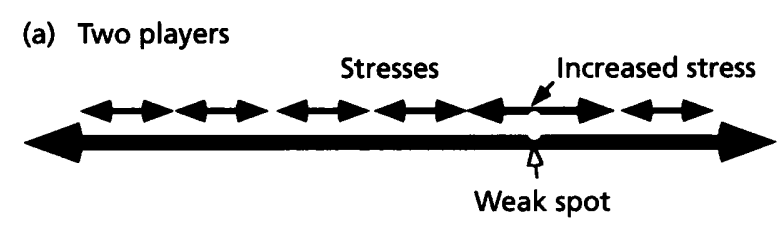

(b) Stress-induced failure

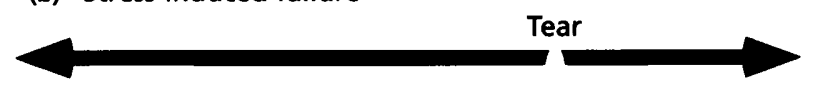

(c) Tug-of-war

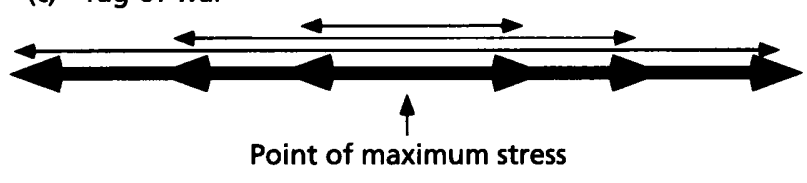

Fig. 1. Development of stress in a stretched body. If a physical body is of uniform cross-section, of a uniform material, and stressed at its two ends, then a uniform tension will be experienced throughout the region in between ( $a$, left side). The magnitude will depend on the forces applied and on the cross-sectional area of the body. If there is a weak spot (a, right side), the stresses may exceed the strength of the body and it may tear (b). If force is applied at a series of points within the object ('tug-of-war'), then the stresses are larger in the centre of the object (c) and this will be the site of rupture even if there is no weak spot. 
engineering mechanics which states that under stress, a bond is more likely to break when adjacent to a defect because at this site the stress is not evenly shared (Griffith, 1920). In Griffith's case, the result of a breakage event is an increase in tension on an adjacent bond and increasing probability of that one breaking. Due to the repetitive transfer of stress to other bonds and their failure, the entire structure fails in the process called 'brittle fracture'.

We suggest that in the case of a living biological structure, a mechanism exists so that increased stresses lead to the triggering of some local safety response that pre-empts the physical destruction of the wall and its weakest component, the CM. These processes would be initiated by a cellular sensory mechanism that responds to a much smaller stress than one that could rupture the covalent bonds of the sacculus or even the non-polar bonds holding the bilayer together. The tension may elicit the response of cell division, which in turn would decrease the tension by a wall growth response acting in an altered cell growth pattern.

Centring due to the 'tug-of-war' principle. Mechanical forces can serve to localize the centre of a structure under special conditions. If instead of the weak spot shown in Fig. 1(a) there was an additional source of tension developed more centrally, then the structure would more probably rip, or trigger a response, in the region where the summation of sources of tension act. Fig. 1(c) shows the results if the stresses are applied in segments along the length of the body. This can be best understood by analogy to a 'tug-of-war' in which two teams grab onto a length of rope and start pulling in opposite directions. Although the stronger team wins, the tension in the rope reaches a maximum between the two teams. This is because a certain amount of tension develops in the rope between the most peripheral players on the two teams, but additional tension is developed between the next two players. Between the next inside pair more tension would develop and so on. If the teams have the same number of equally strong players and were uniformly distributed along the rope, the stress maximum is at the rope's exact physical centre and this is where it will break unless there is a very weak spot elsewhere. This concept is not new in biology: Hershey used the principal to shear DNA into half- and quarter-sized molecules in the days before restriction enzymes. He passed the DNA solution through a fine hypodermic needle. Shear stresses then developed in this tug-of-war fashion so that the different regions were differentially stressed and finally the molecules were pulled apart by forces developed all along the linear doublestranded molecules leading to mechanical breakage of covalent bonds very near the centre.

Centring due to localized stresses. In Fig. 2 a simple experiment is represented: a sheet of rubber was marked with a set of parallel lines (a). When pulled uniformly in the direction normal to the rulings (Fig. $2 \mathrm{~b}$ ), the distance between the parallel lines increases. If pulled only in the centre of the opposite sides the lines become curved and the spacing and the stress is greatest in the central part (Fig. 2c). If pulled only at one single edge (Fig. 2d) then (a)

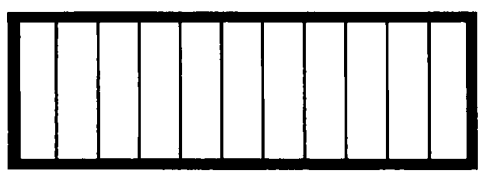

(b)
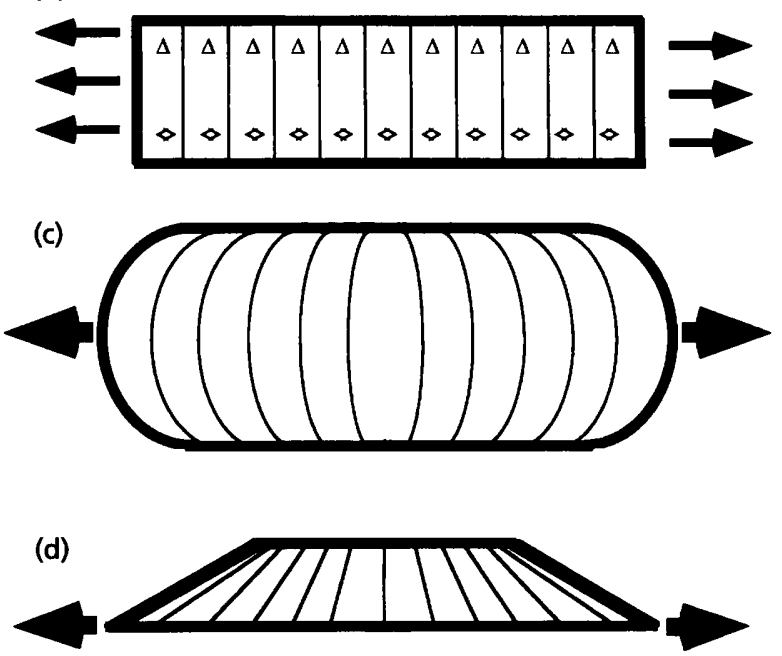

Fig. 2. Development of stress in an elastic sheet. An unstressed body is shown in (a) and one under uniform stress from the ends experiences uniform expansion in (b). It does not matter if expansion results from insertion of new material, shown by the $\Delta s$ or by elastic stress of the old material, shown by the $<>$ symbols. (c) Shows that force applied medially produces greater tension in the centre because the regions on the sides support part of the stress. (d) Shows the same phenomenon of higher tension in the centre for force applied on one edge. In (c) and (d) the maximum stretch and strain occurs at the longitudinal middle.

again the stress is developed on that side and there is a maximum of tension midway between the two forces on the other side. Thus, on the other side the sheet of rubber is not stretched at the free-hanging ends but is stretched near the centre. Basically it is the case outlined in Fig. 2(d) that is important and the basis of the proposed model. But how could the growth of bacteria create a situation that would similarly generate a central peak of stress?

\section{A physical model that could define the central site for cell division}

In this paper we propose a new model (schematically depicted as a mechanical analogy at the bottom of Fig. 3 and a longitudinal sectional view of a cell in Fig. 4) that is based on the differential stresses that would develop between the murein and the underlying CM. Differential stress is build up according to the model because the wall of bacteria is a composite structure in which two layers interact with each other strongly. We will consider only two layers, the murein sacculus and the $\mathrm{CM}$, and therefore neglect the outer membrane possessed by $E$. coli, but not present in $B$. subtilis. Each layer can be mechanically stressed and responds by stretching (and thus becoming 

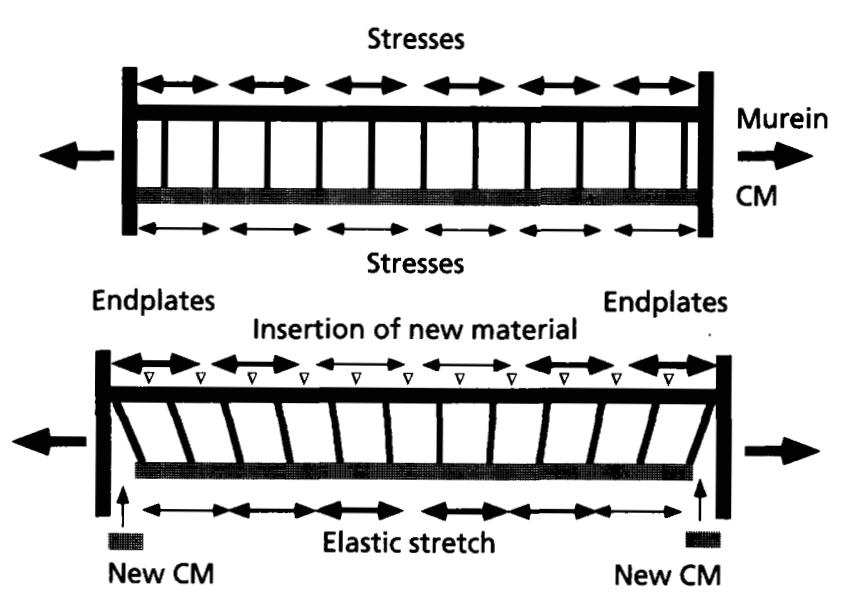

Fig. 3. Development of localized stress in a composite body. At the top, a body composed of an upper murein layer and a lower CM layer attached point by point is stretched at its ends. It does not matter, as in Fig. 2(b), if elongation occurs by stretch or by new incorporation, the stress will be uniform along the length. In the bottom panel, the murein enlarges by insertion of new material as shown by the $\Delta s$ and variable elastic stresses develop in the free-hanging $\mathrm{CM}$ layer. These stresses reach a maximum at the centre. This model is not realistic because there are no gaps in the CM layer of cells, but it illustrates what is needed to make a workable model.

strained), but in addition, in our biological situation each can grow by the insertion of new material and in so-doing become elongated and less stressed. The physical and biochemical properties of the phospholipid and murein layers are quite different. We have not included murein turnover because this seems to be an integral part of 'safe' wall growth (Höltje, 1993). However, we do not exclude differential turnover of phospholipids being involved as suggested by Norris (1992).

The biophysical state of the phospholipid bilayer. Phospholipid bilayers by their nature are two-dimensional objects constrained in one of their three dimensions. Above their freezing point, they behave as nearly incompressible fluids in the other two dimensions. While individual molecules of phospholipid may diffuse, the total area is capable of little expansion $(2-5 \%)$ without tearing or little compression (2-5\%) without buckling (Bloom et al., 1991). This means that they have cohesiveness and if constrained in one surface direction could transmit force to the other. Thus, as the cell grows and elongates at constant diameter, if new phospholipid were not inserted rapidly enough a destructive tension would develop in the CM bilayer. In some cases of bilayer expansion, destruction is prevented by the fusion of extant membrane-bounded vesicles that fuse to the CM, and in some cases of bilayer contraction, such as when the cytoplasmic volume shrinks due to osmotic challenge, vesicles can be formed endocytotically (Kell \& Glaser, 1993; Bloom et al., 1991; Schwarz \& Koch, 1995). Without the introduction of new lipid the $\mathrm{CM}$ would fail in a few seconds during growth of bacteria in rich medium. This, of course, ordinarily does not happen; consequently, the introduction of new membrane
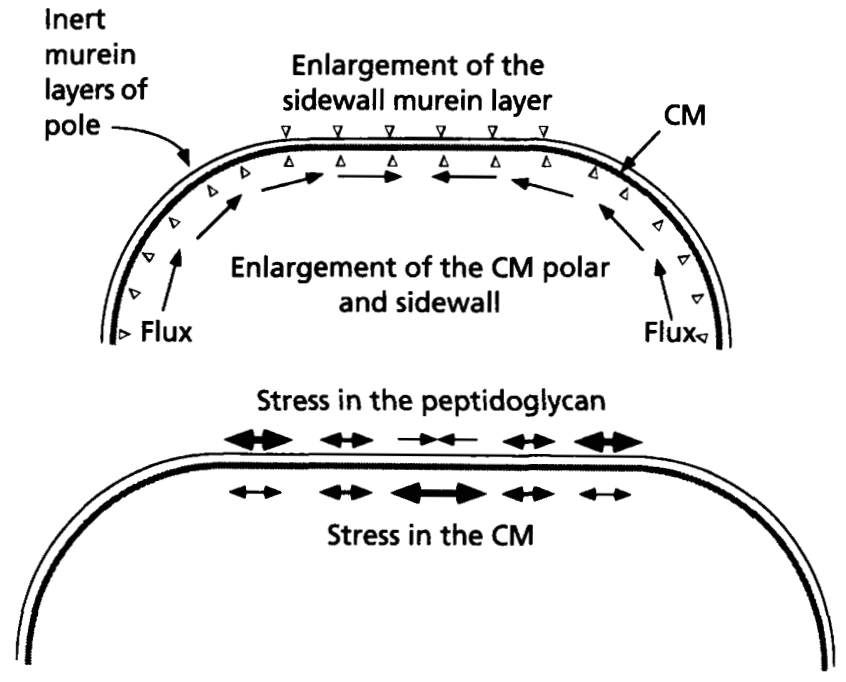

Fig. 4. Central Stress Model. The $\Delta s$ indicate incorporation of murein or new phospholipid molecules into the growing wall. The arrows show the flux of phospholipids towards the centre of the cell wall.

must be fast and is effectively linked to cell elongation. Still, the CM of the growing rod-shaped cell would experience tension over its length if incorporation of phospholipid responded to tension in the CM because of its linkage to the murein layer. It is assumed that phospholipid incorporation into the CM occurs in response to the elongation of the murein and quickly, but belatedly, reduces tension in the CM. In bacteria, in contrast to higher cells, growth of the bilayer is by direct incorporation of phospholipids and not by the creation of vesicles in the Golgi that then fuse with the cell membrane.

The biophysical state of the murein layer. In E. coli, growth and elongation of the peptidoglycan layer of the bacterial sidewall occurs by continuous and diffuse insertion into the murein monolayer. Wall growth occurs by insertion by a system of localized linkages and subsequent cleavages drawing the new material into the stress-bearing plane and permitting safe enlargement (Koch, 1983, 1995a; Höltje, 1993). In B. subtilis, safe growth occurs by the formation of a complete new layer under the old cylindrical wall concomitantly with autolysis of the outermost layer. The new layer in the Gram-positive and the entire layer in the Gram-negative are covalently linked fabrics (see Koch, 1995a, 1988a) capable of a great deal of elastic stretch (Koch \& Woeste, 1992). Thus, both in the murein layer and in the phospholipid layer wall tension develops during cellular growth that is then relieved by murein and phospholipid insertion.

The biophysical state of the $\mathrm{CM}$-murein composite structure. The interaction between the $\mathrm{CM}$ and the murein results because the $C M$ is pressed against the murein due to osmotic forces and in part from membrane proteins that specifically associate with the murein. In particular, these are the membrane-bound penicillin-binding proteins 
(Spratt, 1975) which are involved in the covalent insertion of additional murein subunits into the pre-existing murein sacculus during growth as well other aspects of murein metabolism (see Koch, 1995a). Furthermore, the nascent murein may remain covalently linked for some time to the membrane-residing hydrophobic undecaprenylpyrophosphate carriers that shuffle the murein precursors across the CM (for review see Rogers et al., 1980; Koch, 1995a). Thus osmotic forces, the machinery for murein growth, and the secretion of outer-membrane proteins and exogenous proteins provide a major temporary physical connection between the two layers that would tend to inhibit, but not block, slippage of one layer against the other on the cylindrical part of the cell.

Distribution of tension along the length of the $\mathrm{CM}$-murein complex. As the cell cycle progresses, tensile forces increase in the $\mathrm{CM}$ as murein elongation takes place decreasing the share borne by the murein layer. The balance returns as the $\mathrm{CM}$ subsequently enlarges. A balance is indicated in the upper part of Fig. 3. If the CM response were quick and complete then the entire stress would be borne by the murein layer. If the two layers have the same length as shown by the distance between the endplates, the stresses in each layer would be the same throughout their lengths, no matter where or when elongation takes place, but different for the two. The bottom panel of Fig. 3 shows the mechanical variation that would lead to a central peak of stress in the CM analogously to Fig. 2(d).

The Central Stress Model shown in Fig. 4 shows how the stresses in the CM can become localized in a single peak in the centre of the cell whose magnitude increases as the cell grows. At a sufficient value this localized stress initiates cell division at that site.

The mechanical model shown in Fig. 3 is not plausible because there are no gaps in the CM, but it led to the Central Stress Model as shown in Fig. 4 through the incorporation of additional biological considerations. We have looked through many cryofixed freeze-substituted electron micrographs (Schwarz \& Koch, 1995), and have not seen discontinuities or gaps in either the $\mathrm{CM}$ or the murein layer of the pole. Although in the long run equal areas of new murein and bilayer are added to the wall of the bacteria, differential stresses develop because the timing and topology are different. The essential feature of the model is that it is now known that murein is only inserted in the cylindrical part of the cell and not into a completed pole (Koch \& Woldringh, 1995). Not only the peptidoglycan but also the outer-membrane poles of $E$. coli are metabolically inert (Koch \& Woldringh, 1995). However, there is no evidence to suggest that phospholipid is not inserted into the $\mathrm{CM}$ of an established pole. It is reasonable to assume, therefore, that the new lipid is inserted all over the bilayer, including the poles. Although experiments have been published showing that the peptidoglycan and membrane incorporation occur in parallel amounts (Gally et al., 1993), this only shows that as the wall invaginates equal surface areas of bilayer and murein must be formed, and it is not relevant to the question of whether local differential syntheses of $\mathrm{CM}$ and murein exist as we propose. Thus we assume that there is continuing flow of bilayer material from the old poles into the cylindrical region.

\section{Triggering of cell division}

The Central Stress Model assumes that once the CM stress threshold is exceeded at any place on the cell wall, a local response occurs. One possibility is that a special class of membrane-bound molecules experience an irreversible change due to this tension. These may be the sensor component of a yet unknown specific two-component system or something analogous. Ordinarily the response is localized at a circumferential circle around the middle of the cell at its exact centre. We imagine that this response speeds the local wall growth and fosters centripetal growth. Through this enlargement, the turgor pressure is decreased, preventing any additional site (such as at the junction of cylinder and an old pole) triggering. It is assumed that such dedicated two-component systems are present and monitoring the tension of the phospholipid layer in all portions of the cell membrane. Twocomponent systems exist for dozens of functions in prokaryotes. Specifically, there is a system for osmoregulation that meets the postulated specifications (see Stock et al., 1989; Surette \& Stock, 1994). The general pattern of two-component systems is that on being stimulated the sensory elements cause the sensor elements to become autophosphorylated. Subsequently, the phosphate is transphosphorylated to the effector component that elicits transcription, leading to the synthesis of special proteins that implement cell division. (An alternative possibility to a role for two-component system is a response due to stress-sensitive channels.)

The model proposed here also attributes to the sensory component responding to membrane tension two additional functions that are not known for the other twocomponent systems. One is that the membrane-bound component, when triggered, changes itself irreversibly and the signal remains in place as a marker of a future cell division site. This could likely be done if the autophosphorylation in some cases did not lead to transphosphorylation. The second function is that triggered molecules serve to bind other components to lead to the development of cell division. Local responses are not characteristic of known two-component systems, and therefore we are assuming a novel additional feature for such systems. It may be relevant that FtsA is unphosphorylated in the membrane and phosphorylated (Sanchez et al., 1994) while in the cytoplasm, but it can be suggested that those molecules in the membrane at the site of a future cell division are phosphorylated. Several suggestions can be made for the trigger action. Perhaps the initiating cell division events are mediated by the sensor in its conformationally altered shape causing changes in the phospholipid composition of the bilayer (Cullis \& De Kruijff, 1979; Gruner, 1985; De Kruijff, 1987; Bloom et al., 1992; Norris, 1992). Perhaps it may result from the localized binding of newly synthesized division proteins. More probably it could be that the sensor portion of the hypothetical two-component system 


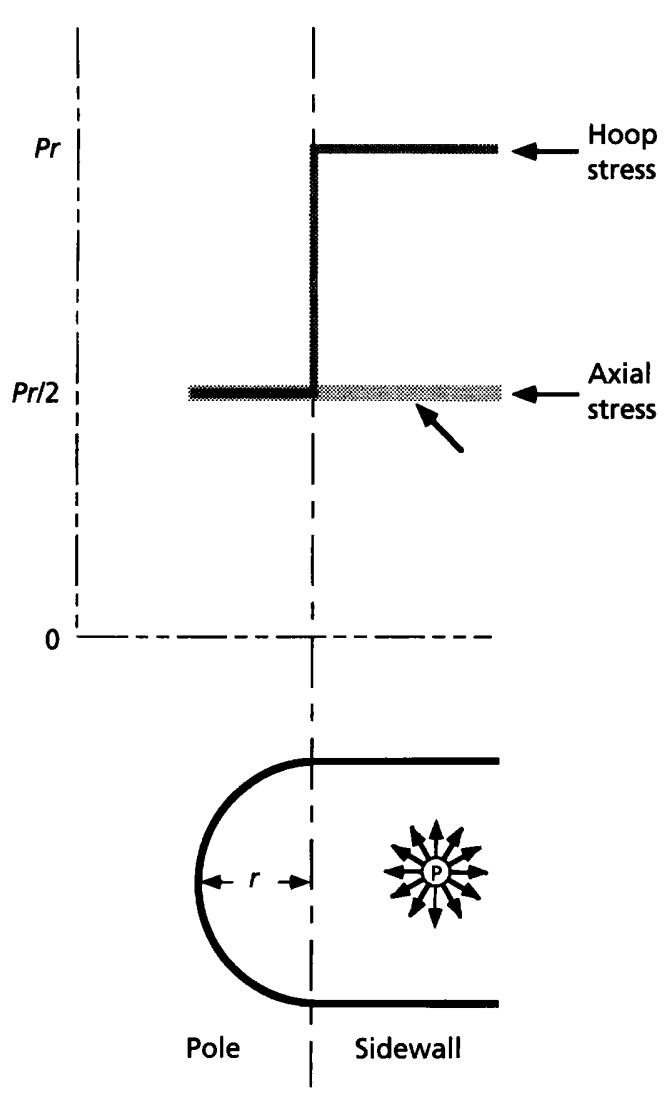

Fig. 5. Discontinuity of stresses at the junction of the pole and the sidewall. The lower panel shows an idealized cylindrical cell with a hemispherical pole. The stresses developing in the wall are shown in the top panel. The axial (or meridional) stress is shown by a thicker grey line; the hoop (or circumferential) stress is shown by the thinner grey line. The axial stress is the same in both regions of the cell while the magnitude of hoop stress doubles abruptly in passing from the pole to the cylindrical region. It is suggested that under certain circumstances when normal division in the centre of the cell is blocked, this discontinuity in circumferential stress serves to trigger the assumed two-component system to initiate cell division. In this case division creates a minicell.

causes the FtsZ protein to accumulate (Ward \& Lutkenhaus, 1985) as a ring going circumferentially around the cell at the site where the threshold of the sensory system had been exceeded. Interestingly, filamentous cells blocked in cell division start dividing at pre-determined sites when returned to conditions that allow cell division (Donachie et al., 1984). This is consistent with the proposal that the localized sites had been marked by phosphorylation or other kinds of changes (say dislocation of the CM from the outer membrane) and the triggering event remembered, despite a block in immediate expression of that event. This observation suggests that there must be some way to 'freeze' the postulated sensor for cell division in a changed but temporarily inactive conformation. One could speculate that what happens is that the signal blocks murein synthesis locally but not bilayer synthesis. In the wild-type strain, murein synthesis would not be blocked, but be altered to engage in the constriction process. In the mutant this block of murein incorporation would mean that as the sidewall elongated the centre between the blocked division sites would be found by the mechanism proposed above and thus generate a series of marked presumptive sites for cell division. It must be noted that normal chromosome replication is completed before the invagination for cell division can be first detected, but completion is not necessary for division in a variety of circumstances, for example during minicell formation. Consequently, the proposed mechanism need not be directly connected to chromosomal replication.

\section{Formation of minicells}

In addition to a local maximum in one layer and a minimum in the other at the cell's centre, created as a result of differential growth of the two layers of the cell wall, there is also a permanent discontinuity at the junction of the hemispherical polar caps with the cylindrical part of the cell wall (Koch, 1988a) (Fig. 5). At this location there is a twofold increase in one of the two orthogonal stress components (Koch, 1988a). The circumferential, or hoop, component of the surface stress in the cylindrical region, if $P$ is the pressure and $r$ is the radius of the cell, is $P r$, while that in the pole is $\operatorname{Pr} / 2$, and consequently there is an abrupt twofold discontinuity. For the meridional, or axial, component the stress is equal to $\mathrm{Pr} / 2$. So there is no discontinuity of axial stress at the junction in this component of stress. (These expressions assume that both wall regions are homogeneous, made of the same material, and are of the same unit thickness. It is also assumed that the pole is hemispherical; the discrepancy between the two stresses would be less for a prolate pole and more for a oblate pole. Poles of Gram-negative cells are oblate as formed.) Assuming that the murein supports only part of this stress and that some portion of the stress is transferred to the $\mathrm{CM}$, then the tension would change very significantly over a very small region of membrane. Thus, in a rod-shaped bacterium there is a second site where there is a discontinuity in a component of the stress.

Under a variety of conditions bacteria do divide inappropriately at the junction region and produce small round DNA-less cells, called minicells (Adler et al., 1967; Frazer \& Curtiss, 1974). This outcome can be favoured by mutations in $\min C D$ or in $\min E$ (de Boer et al., 1989), but it can also be engineered by overproduction of the $f t s Z$ gene product (Ward \& Lutkenhaus, 1985). In the model proposed here, this kind of error can be understood on the basis that in these mutants (as in normal cells) the twocomponent system is distributed in all parts of the cell's bilayer. These can be stimulated not only at the centre of the cell but also at the poles because of the local discontinuity of stress when these sites are no longer under the veto of the $\min C D$ cell division inhibitor.

\section{The relationship between evenness of division and maintenance of synchrony}

Bacterial cells divide more directly in response to their achieving a critical biomass and less directly to their 
achieving a particular age (Schaechter et al., 1962; Koch \& Schaechter, 1962). Thus, timing of cell division is much less relevant than the cell's success in carrying out metabolism. It follows that if four criteria are obeyed, synchrony would persist (Koch \& Schaechter, 1962). Although some synchrony would be lost after the first division, a partial synchrony would be maintained indefinitely if four conditions were met. They are: (i) full synchrony is initially achieved; (ii) division occurs at a particular size; (iii) cell division always partitions the cell into equal sized daughters; (iv) conditions are maintained so that biochemical processes proceed at the same specific rate in all cells in the population. Maintenance of synchrony results from the cellular mechanism that triggers cell division at the critical biomass for the strain and growth conditions. Then those cells at division which happen to vary by chance either above or below the mean division size would vary in the time to the subsequent division in the opposite direction; thus a smaller than average new-born cell would take longer than average to reach the mean size for division. Consequently, the total variation would not accumulate unless there was an inheritance of size-at-division. Thus, the net effect of fluctuations in division size during successive divisions cancels out (Koch, 1966, 1995b; Koch \& Schaechter, 1962, but see Cooper, 1991, pp. 213-234). Experimental confirmation was obtained (Koch, 1966) through the analysis of the growth of single cells into microcolonies from the data of Hoffman \& Franks (1965) and the cell pedigrees observed by Kubitschek (1962). Subsequently, other experiments were reported in which mass cultures maintained synchrony while growing in constant conditions in dilute suspension. This was shown by Marjorie Kelley (see Koch, 1977) and by Cutler \& Evans (1966). Kepes \& Kepes (1981) also prepared cultures that exhibited a large number (sometimes as many as 15) of synchronous division steps. These reports of persistent synchrony are consistent with the idea that all the criteria mentioned above were rigorously obeyed in those cases. The relevance here is that these examples of persistent synchrony are evidence that cell division can produce very nearly equally sized daughter cells. Probably the average unevenness in division is even less than the experimental estimate drawn from electron microscopic measurements (see Trueba, 1981; Grover et al., 1987; Koppes et al., 1987 ) in which for one strain there was only a $4 \% \mathrm{CV}$ in the ratio of the birth to mother sizes of an $E$. coli culture. Consequently, even though the persistence of long-term synchrony has been demonstrated only rarely, probably because it is difficult to meet all four criteria simultaneously, it has been demonstrated widely enough that it can be considered an established fact and puts a high importance for subscribing only to models in which division of the mother generates two equal-sized daughters. Note that this last statement represents an unpopular view (see Cooper, 1991, pp. 35 \& 37), but the negative results of failure to establish long-term synchrony in the many experiments of Helmstetter and of Cooper with the membrane elution technique does not exclude the possibility of generating synchronous cultures.

\section{Relationship of cell division to DNA replication}

Descriptions of the bacterial cell cycle are usually based on the Helmstetter \& Cooper (1968) analysis. Their hypothesis posits that the time needed for completion of chromosome replication, $C$, and the subsequent period of time until cell division is complete, $D$, are well-controlled by cell physiological processes. But this formulation leaves unspecified how the duration of $I$ is determined. $I$ is the time between subsequent initiations of chromosome replication and therefore is also equal to the doubling time of the bacteria. The idea in the Helmstetter \& Cooper (1968) model is that it is the time to accumulate a unit amount of the initiator for chromosome replication. Clearly this time depends on the rate of synthesis which, in turn, depends on the nutritional state of the cell and brings us back to the point that the cell division cycle depends directly on rates of synthesis and less directly on the time since the last initiation. The studies of Schaechter et al. (1962) showed that the cell appears to respond to its own success in achieving critical sizes at both cellular division and nuclear division. This was further emphasized by Donachie (1968), who demonstrated that the cell seems to trigger some critical event, probably chromosome replication, at a particular cell mass, independently of the growth rate. This older work seems to be connected to DnaA-binding sites in the oriC region and to sites of methylation of the adenine residues of DNA (see von Meyenburg \& Hansen, 1987). Studies with a flow cytometer (Boye \& Lobner-Olesen, 1991) have shown that initiation of sister chromosomes in a common cytoplasm occurs synchronously. In spite of these great successes, no studies so far have found a biochemical measuring system that could allow the cell to estimate its own biomass and enable it to initiate cell division at the appropriate stage in the cell's growth. We now suggest that the trigger responds directly to the peak tension in the $\mathrm{CM}$ and this not only leads to a central position for cell division, but it coordinates cell division directly with cell length and less directly with current biomass and still more indirectly with the accumulated age.

\section{Conclusions}

The tug-of-war analogy of Fig. 1(c), suggests that extra sources of axial tension in the middle of the cell could lead to a special event at the cell's middle. The longitudinal force on the cell cylinder derives from the hydrostatic pressure on the poles. A possible way in which tension in the CM could peak in the centre is if peptidoglycan insertion takes place predominantly towards the centre of the cell and not just as part of the next cell division event. This is a possible interpretation from the work of Stephen Cooper (Ann Arbor, MI, USA; personal communication) and Mulder \& Woldringh $(1989,1993)$. Similarly, special properties of the distribution of phospholipid could be invoked (Norris, 1992). This could lead to a central decrease of tension in the murein layer. Since, however, the total tension along the composite wall must be constant, the decrement would be transferred to the CM. Such a situation would only help to locate the centre if there was some other mechanism to favour insertion in 
the centre; but then the argument is circular. This problem is obviated by postulating a flow of bilayer material from the poles.

Is there a necessity for evenness of division? Many bacteria, and certain E. coli strains under certain conditions, do not divide to create equal-sized daughters. So it is an anomaly that bacterial division in certain cases can be very precise. It is suggested that this precision in the evenness of division in the creation of a pair of daughter cells reflects that the cell's method of the fixation of its centre is inherently precise due to physical laws. In this case it may be easier to be precise than to be sloppy. It may be that the precision is not needed for evenness of the cell division process, but for the linkage of adequate cell growth with the initiation of chromosome replication and of cell division.

The major impetus to developing the Central Stress Model is that in spite of many years of study of the bacterial cell division process, we still do not understand how the cell measures its own success in biomass production to 'know' when to divide and at what location it should start constricting. Physiology, ultrastructural research, and molecular biology have led to suggestions but not to an understanding of the mechanism that the cell employs. Search for mutants has also failed. This failure and the fact that cell division can sometimes, but not always, be highly precise suggested that a mechanism functions as proposed here which does not depend on genes directly but instead depends on simple physical circumstances.

\section{ACKNOWLEDGEMENTS}

We thank Uli Schwarz for his encouragement and support, Hans Meinhardt for helpful criticism, Yi Chen for demonstrating a model system in which tensile stress in gels covalently bound to the wall of capillaries leads to the even distribution of voids within the gel, Jürgen Lommatzsch for his thoughts about minicell formation, Markus Templin for taming the unruly jungle of laboratory computers, and Heinz Schwarz for electron micrographs. We would also like to thank Stephen Cooper and Vic Norris for extremely stimulating suggestions.

\section{REFERENCES}

Adler, H. I., Fisher, W. D. Cohen, A. \& Hardigree, A. A. (1967). Miniature Escherichia coli cells deficient in DNA. Proc Natl Acad Sci US $A$ 57, 321-326.

Bloom, M., Evans, E. \& Mouritsen, O. G. (1991). Physical properties of the fluid lipid-bilayer components of the cell membrane: a perspective. $Q$ Rev Biophys 24, 293-397.

de Boer, P., Crossley, R. E. \& Rothfield, L. I. (1989). A division inhibitor and a topological specificity factor coded for by the minicell locus determine proper placement of the division septum in E. coli. Cell 56, 641-649.

Boye, E. \& Lobner-Olesen, A. (1991). Bacterial growth control, studied by flow cytometry. Res Microbiol 142, 131-135.

Burdett, I. D. J. \& Higgins, M. H. (1978). Study of pole assembly in Bacillus subtilis by computer reconstruction of septal growth zones seen in central longitudinal thin sections of cell. J Bacteriol 133, 959-971.
Cook, W. R. \& Rothfield, L. I. (1994a). Early stages in development of the Escherichia coli cell-division site. Mol Microbiol 14, 485-495.

Cook, W. R. \& Rothfield, L. I. (1994b). Development of the celldivision site in FtsA ${ }^{-}$filaments. Mol Microbiol 14, 497-503.

Cook, W. R., MacAlister, T. J. \& Rothfield, L. I. (1986). Compartmentalization of the periplasmic space at division sites in gram-negative bacteria. J Bacteriol 168, 1430-1438.

Cook, W. R., Kepes, F., Joseleau-Petit, D., MacAlister, T. J. \& Rothfield, L. I. (1987). Proposed mechanism for the generation and localization of new division sites during the division cycle of Escherichia coli. Proc Natl Acad Sci US A 84, 7144-7148.

Cook, W. R., de Boer, P. A. J. \& Rothfield, L. I. (1989). Differentiation of the bacterial cell division site. Int Rev Cytol 118, 1-31.

Cooper, S. (1991). Bacterial Growth and Division. San Diego: Academic Press.

Cullis, P. R. \& De Kruijff, B. (1979). Lipid polymorphism and the functional roles of lipid in biological membranes. Biochim Biophys Acta 559, 399-420.

Cutler, R. G. \& Evans, J. E. (1966). Synchronization of bacteria by a stationary-phase method. $J$ Bacteriol 91, 469-476.

De Kruijf, B. (1987). Polymorphic regulation of membrane lipid composition. Nature 329, 587-588.

Donachie, W. D. (1968). Relationship between cell size and the time of initiation of DNA replication. Nature 219, 1077-1079.

Donachie, W. D., Begg, K. J. \& Sullivan, N. F. (1984). The morphogenes of Escherichia coli. In Microbial Development, pp. 27-62. Edited by R. Losick \& L. Shapiro. Cold Spring Harbor, NY : Cold Spring Harbor Laboratory.

Frazer, A. \& Curtiss, R. (1974). Production, properties and utility of bacterial minicells. Curr Top Microbiol Immunol 69, 1-84.

Gally, D., Bray, K. \& Cooper, S. (1993). Synthesis of peptidoglycan and membrane during the division cycle of rod-shaped, gramnegative bacteria. $J$ Bacteriol 175, 3121-3130.

Grover, N. B., Woldringh, C. L. \& Koppes, L. J. H. (1987). Elongation and surface extension of individual cells of Escherichia coli $\mathrm{B} / \mathrm{r}$ : comparison of theoretical and experimental size distributions. $J$ Theor Biol 129, 337-348.

Gruner, S. M. (1985). Intrinsic curvature hypothesis for biomembrane lipid composition: a role for nonbilayer lipids. Proc Natl Acad Sci US A 82, 3665-3669.

Griffith, A. A. (1920). The phenomena of rupture and flow in solids. Philos Trans $\mathrm{R}$ Soc Lond Ser A 221, 163-198.

Helmstetter, C. E. \& Cooper, S. (1968). DNA synthesis during the division cycle of rapidly growing Escherichia coli B/r. J Mol Biol 31, 507-518.

Hoffman, H. \& Franks, M. E. (1965). Time-lapse photomicrography of cell growth and division in Escherichia coli. J Bacteriol 89, 212-216.

Holtje, J.-V. (1993). The three-for-one model. In Bacterial Growth and Lysis: Metabolism and Structure of the Bacterial Sacculus, pp. 419-426. Edited by M. A. de Pedro, J.-V. Höltje \& W. Löffelhardt. New York: Plenum Press.

Kell, A. \& Glaser, R. W. (1993). On the mechanical and dynamic properties of plant cell membranes: their role in growth, direct gene transfer and protoplast fusion. $J$ Theor Biol 160, 41-62.

Kepes, F. \& Kepes, A. (1981). Long-lasting synchrony of the division of enteric bacteria. Biochem Biophys Res Commun 99, 761-767.

Koch, A. L. (1966). On evidence supporting a deterministic process of bacterial growth. $J$ Gen Microbiol 43, 1-5.

Koch, A. L. (1977). Does the initiation of chromosome replication regulate cell division? Adv Microb Pbysiol 16, 49-98. 
Koch, A. L. (1983). The surface stress theory of microbial morphogenesis. Adv Microb Pbysiol 24, 301-366.

Koch, A. L. (1985). Primeval cells : possible energy generating and cell-division mechanisms. J Mol Evol 21, 270-277.

Koch, A. L. (1988a). Biophysics of bacterial wall viewed as a stressbearing fabric. Microbiol Rev 52, 337-353.

Koch, A. L. (1988b). Specuations on the growth strategy of prosthecate bacteria. Can J Microbiol 34, 390-394.

Koch, A. L. (1990). Positioning of the cell division site. Res Microbiol 141, 136-139.

Koch, A. L. (1993). Biomass growth rate during the cell cycle. $C R C$ Crit Rev Microbiol 19, 17-42.

Koch, A. L. (1995a). Bacterial Growth and Form. New York: Chapman \& Hall.

Koch, A. L. (1995b). The default growth strategy of bacteria. Third International Conference on Mathematical Population Biology, Pau, France, June 1-5, 1995.

Koch, A. L. \& Schaechter, M. (1962). A model for statistics of the cell division process. J Gen Microbiol 29, 435-454.

Koch, A. L. \& Woeste, S. (1992). The elasticity of the sacculus of Escherichia coli. J Bacteriol 174, 4811-4819.

Koch, A. L. \& Woldringh, C. L. (1995). The inertness of the poles of a Gram-negative rod. $J$ Theor Biol 171, 415-425.

Koch, A. L., Mobley, H. L. T., Doyle, R. J. \& Streips, U. N. (1981). The coupling of wall growth and chromosome replication in Grampositive rods. FEMS Microbiol Lett 12, 201-208.

Koppes, L. J. H., Grover, N. B. \& Woldringh, C. L. (1987). Predicted steady state size distributions for various growth models. $J$ Theor Biol 129, 325-326.

Kubitschek, H. E. (1962). Normal distribution of cell generation rates. Exp Cell Res 26, 439-450.

MacAlister, T. J., MacDonald, B. \& Rothfield, L. I. (1983). The periseptal annulus: an organelle associated with cell division in Gram-negative bacteria. Proc Natl Acad Sci US A 80, 1372-1376.

Meinhardt, H. (1982). Models of Biological Pattern Formation. London: Academic Press.

von Meyenburg, K. \& Hansen, F. G. (1987). Regulation of chromosomal replication. In Escherichia coli and Salmonella typhimurium: Cellular and Molecular Biology, pp. 1555-1577. Edited by F. C. Neidhardt, J. L. Ingraham, K. Brooks Low, B. Magasanik, M. Schaechter \& H. E. Umbarger. Washington, DC: American Society for Microbiology.

Mulder, E. \& Woldringh, C. L. (1989). Actively replicating nucleoids influence positioning of division sites in Eschericbia coli filamentforming cells lacking DNA. J Bacteriol 171, 4303-4314.

Mulder, E. \& Woldringh, C. L. (1993). Plasmolysis bays in Eschericbia coli: are they related to development and positioning of division sites? J Bacteriol 175, 2241-2247.
Norris, V. (1992). Phospholipid domains determine the spatial organisation of the Escherichia coli cell cycle: the membrane tectonics model. $J$ Theor Biol 154, 91-107.

Ogden, G. B., Pratt, M. J. \& Schaechter, M. (1988). The replicative origin of the Eschericbia coli chromosome binds to the membranes only when hemimethylated. Cell 54, 127-135.

Rothfield, L. I., Cook, W. R. \& de Boer, P. A. (1991). Biogenesis of the Escherichia coli cell division system. Cold Spring Harbor Symp Quant Biol 56, 752-756.

Rogers, H. J., Perkins, A. R. \& Ward, J. B. (1980). Microbial Cell Walls and Membranes, pp. 261-264. London: Chapman \& Hall.

Sanchez, M., Valencia, A., Ferrándiz, M.-H., Sander, C. \& Vicente, M. (1994). Correlation between the structure and biochemical activities of FtsA, an essential cell division protein of the actin family. EMBO J 13, 4919-4925.

Schaechter, M., Williamson, J. P., Hood, J. R., Jr \& Koch, A. L. (1962). Growth, cell and nuclear divisions in some bacteria. J Gen Microbiol 29, 421-434.

Schwarz, H. \& Koch, A. L. (1995). Phase and electron microscopic observations of osmotically induced wrinkling and the role of endocytotic vesicles in the plasmolysis of the Gram-negative cell wall. Microbiology 141, 3161-3170.

Spratt, B. G. (1975). Distinct penicillin binding proteins involved in the division, elongation, and shape of Eschericbia coli K12. Proc Natl Acad Sci US A 72, 2999-3003.

Stock, J. B., Ninfa, A. J. \& Stock, A. (1989). Protein phosphorylation and regulation of adaptive responses in bacteria. Microbiol Rev 53, 460-490.

Surrette, M. G. \& Stock, J. B. (1994). Transmembrane signal transducing proteins. In Bacterial Cell Wall, pp. 465-484. Edited by J.-M. Ghuysen \& R. Hakenbeck. Amsterdam: Elsevier.

Trueba, F. J. (1981). A morphometric analysis of Escherichia coli and other rod-shaped bacteria. $\mathrm{PhD}$ thesis, University of Amsterdam.

Van Helvoort, L. M., Joop, M. L. \& Woldringh, C. L. (1994). Nucleoid partitioning in Escherichia coli during steady state growth and recovery from chloramphenicol treatment. Mol Microbiol 13, 577-583.

Ward, J. E., Jr \& Lutkenhaus, J. (1985). Overproduction of FtsZ induces minicell formation in E. coli. Cell 42, 941-949.

Woldringh, C. L. (1994). Significance of plasmolysis spaces as markers for periseptal annuli and adhesion sites. Mol Microbiol 14, 597-607.

Woldringh, C. L., Mulder, E., Huls, P. G. \& Vischer, N. (1991). Toporegulation of bacterial division according the nucleoid occlusion model. Res Microbiol 142, 309-320.

Received 17 May 1995; accepted 11 August 1995. 